\title{
Treatment of advanced melanoma
}

\author{
Van Anh Nguyen (D)
}

Over the past decade the treatment of advanced melanoma has been revolutionized by the approval of new therapeutic agents, including BRAF (v-raf murine sarcoma viral oncogene homolog B1) and MEK (Mitogen-activated protein kinase) inhibitors, cytotoxic T-lymphocyte-associated antigen 4 (CTLA4) and programmed cell-death protein 1 (PD-1) blocking antibodies, as well as a modified oncolytic herpes virus that is given intratumourally. These changes in the treatment landscape have dramatically improved objective response rates, progression-free survival and overall survival for patients. Specifically, the overall survival of patients with metastatic melanoma has improved from 9 months before 2011 to ongoing longterm tumour control in a subset of patients.

However, despite the major advances that have been achieved, the management of advanced melanoma remains challenging as responses to treatment are heterogeneous and not always durable. Hence, additional improvements are clearly required, and several strategies are in clinical development with novel agents in the pipeline. Various combination strategies, combining different immunotherapies with one another (e.g., anti-Lymphocyte-activation gene 3 [LAG-3], pegylated Interleukin-2 [IL-2], Toll-like receptor 9 [TLR-9] agonists, anti-glucocorticoid-induced TNFR-related protein [GITR]) as well as with others (e.g., BRAF/MEK inhibition, intralesional drugs, Histone deacetylases [HDAC] inhibitor, vaccines), mostly in combination with a PD-1 backbone, are being investigated with promising results. Alternative sequencing strategies are also under investigation.

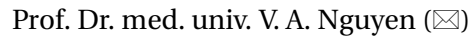

Department of Dermatology, Venerology and

Allergology, Medical University of Innsbruck,

Anichstraße 35, 6020 Innsbruck, Austria

Van.Nguyen@i-med.ac.at
These new approaches will provide additional evidence to guide treatment decisions, increase systemic treatment options and improve long-term outcomes for patients with advanced melanoma. Moreover, the identification of biomarkers that can predict patient benefits from specific treatment strategies may help to improve patient outcomes.

Following the successes with immunotherapies and targeted therapies for metastatic melanoma, these agents have also been evaluated in the adjuvant setting in patients with locally advanced melanoma at high risk for recurrence, with the goal of eliminating residual microscopic disease to improve relapse-free and overall survival. The field is now shifting towards implementing systemic therapies in the neoadjuvant setting for bulky, clinically detected disease. Neoadjuvant therapy has several potential advantages. In particular, it can limit surgical morbidity and allows insights into tumour biology and treatment response. First results from neadjuvant trials are very promising and may change the paradigm for some patients. Again, research and biomarkers to characterize the risk of recurrence may help to individualize treatment decisions regarding the use of a neoadjuvant therapy versus surgery followed by adjuvant therapy.

The therapeutic options for patients with melanoma are rapidly evolving. In future, we must continue to develop more patient-centered treatment that will provide maximum benefit and minimal risk to individual patients.

The articles presented in this issue of memo are intended to give an overview on the most important aspects of the treatment of advanced melanoma [1-3].

Van Anh Nguyen 


\section{editorial}

Conflict of interest V.A. Nguyen has received speaker's honoraria and consultancy fees from Amgen, Bristol- Myers Squibb, GlaxoSmithKline, Merck Sharp \& Dohme, Novartis, Roche and Takeda outside the submitted work.

\section{References}

1. Koelblinger P. Adjuvant and neoadjuvant treatment of melanoma. memo. 2020;13(3). https://doi.org/10.1007/ s12254-020-00602-3.

2. Richtig E. The therapeutic landscape of advanced melanoma. memo. 2020;13(3). https://doi.org/10. 1007/s12254-020-00593-1.

3 . Hoeller Ch. The future of combination therapies in advanced melanoma. memo. 2020;13(3). https://doi.org/10. 1007/s12254-020-00640-x.
Publisher's Note Springer Nature remains neutral with regard to jurisdictional claims in published maps and institutional affiliations.

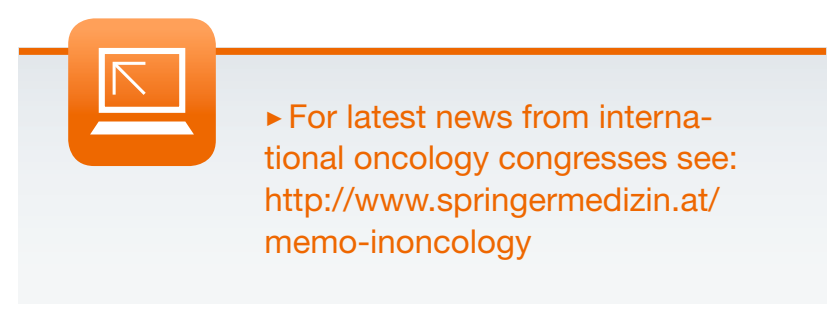

